\title{
Fluctuation-dissipation ratio in lattice-gas models with kinetic constraints
}

\author{
Mauro Sellitto \\ Dipartimento di Scienze Fisiche and Unità INFM, \\ Università "Federico II", Mostra d'Oltremare, Pad. 19, I-80125 Napoli, Italy \\ Mauro.Sellitto@na.infn.it \\ and \\ Instituto de Física, UFRGS, CP 15051 \\ 91501-970 Porto Alegre RS Brazil
}

(July 30, 2018)

\begin{abstract}
We investigate by Montecarlo simulation the linear response function of three dimensional structural glass models defined by short-range kinetic constraints and a trivial equilibrium Boltzmann-Gibbs measure. The breakdown of the fluctuation-dissipation theorem in the glassy phase follows the prediction of mean field low temperature mode-coupling theory.
\end{abstract}

05.20.-y, 64.70.Pf, 61.20.Ja

Introduction. Glassy materials are a paradigmatic example of systems characterized by slow relaxation and physical aging phenomena [1,2]. While thermodynamic observables are rather regular along the glass transformation, dynamical properties undergo drastic changes, and in particular relaxation times increases up to 15 orders of magnitude. The microscopic origin of such a dramatic slowing down is the so called cage effect: a tagged particle in a liquid does not perform a simple random walk but rattles in a cage formed by the surrounding particles, and moves away from it only if the particles building up the cage walls are themselves able to move. At low temperature or high density, when the degrees of freedom of the particles become strongly coupled, this cooperative process exceeds observation time and the glass appears like a "solid" [3].

However, structural relaxation is not completely absent in glasses, and molecular rearrangements still occur on longer time scales. Indeed, after a quench in the low temperature or high density phase, particle diffusion does not stop altogether, but rather becomes slower and slower as time goes on. This is the origin of aging effect: the response to an external perturbation depends on the "age" of the sample, i.e., on the time elapsed since its preparation, even at very long times. Therefore in this regime glassy systems never reach thermodynamic equilibrium and, consequently, time translational invariance (TTI) and the fluctuation-dissipation theorem (FDT) are violated.

The problem. Despite the remarkable progress realized in the study of out-of-equilibrium dynamics of aging systems [- [4], the detailed nature of ergodicity breaking in finite-dimensional glasses is not yet fully understood. In mean-field mode-coupling theory the glass transition appears as a purely dynamic effect due to an instability of the equation governing the correlation of density fluctuations [3]. In particular, mean-field disordered models of structural glasses show that the origin of this dynamical transition is the existence of a large number of metastable states which trap the system for an infinite time. On the other hand, the life-time of metastable states in finitedimensional short range models is finite, since it is always possible to nucleate, by a thermally activated process, a droplet of the stable phase. Therefore the dynamical transition appear as an artifact of the mean-field approximation, and in real glasses this transition would be a finite-time effect, at least on time scales much smaller than the life-time of metastable states [5 8 ].

However, since salient features of glassy behavior are essentially of dynamical nature, and since dynamical universality classes are smaller than the static ones, it is important to establish how glassy effects depend on the details of dynamics. In this paper we explore the limit case of three dimensional lattice gas models defined only by short-range kinetic constraints and by a trivial equilibrium measure, in which the glass transition appears to have a purely dynamical origin unrelated to the existence of metastable states 6, 10. Previous Montecarlo simulations have shown that this model reproduces qualitatively the "glassy" phenomenology, including annealingrate dependence, irreversibility effects, and waiting-time dependent logarithmic diffusion 10,11. Here we investigate aging effects in the linear response function in order to characterize the violation of FDT [12,13].

The model. Our starting point is the kinetic latticegas model introduced by Kob and Andersen in order to mimic the cage effect in supercooled liquids [9]. The system consists of $N$ particles in a cubic lattice of size $L^{3}$, with periodic boundary conditions. There can be at most one particle per site. Apart from this hard-core constraint there are no other static interactions among the particles. At each time step a particle and one of its neighbouring sites are chosen at random. The particle moves if the three following conditions are all met:

1. the neighbouring site is empty; 
2. the particle has less than 4 nearest neighbours;

3. the particle will have less than 4 nearest neighbours after it has moved.

The rule is symmetric in time, detailed balance is satisfied and the allowed configurations have the same weight in equilibrium. With this simple definition one can proceed to study the dynamical behavior of the model at equilibrium. One observes that the dynamics becomes slower and slower as the particle density $\rho$ increases; in particular, the diffusion coefficient of the particles, $D$, vanishes as the density $\rho$ approaches the critical value $\rho_{\mathrm{c}} \simeq 0.88$, with a power law

$$
D(\rho) \sim\left(\rho_{c}-\rho\right)^{\phi},
$$

with an exponent $\phi \simeq 3.19$.

Since we are interested in the dynamical approach to the putative equilibrium state we allow the system to exchange particles with a reservoir characterized by a chemical potential $\mu$. Therefore, we alternate the ordinary diffusion sweeps with sweeps of creation/destruction of particles on a single layer with the following Montecarlo rule: we randomly choose a site on the layer; if it is empty, we add a new particle; otherwise we remove the old particle with probability $\mathrm{e}^{-\beta \mu}$. The number of particles is no longer fixed and the external control parameter is $\mu$, which plays the role of the inverse temperature. In this way we can prepare the system in a non equilibrium state by a process analogous to a quench, which is represented by a jump in $1 / \mu$ from above to below $1 / \mu_{c}$ (where $\mu_{c}$ is defined through the state equation $\left.\rho\left(\mu_{c}\right)=\rho_{c}\right)$. The situation becomes analogous to the canonic case in which one controls the temperature, and the energy endeavors to reach its equilibrium value.

Linear response. Following a suggestion by J. Kurchan, we compute the linear response function by applying to our system a small random perturbation at time $t_{w}$ :

$$
\mathcal{H}_{\epsilon}=\epsilon \sum_{a=1}^{3} \sum_{k=1}^{N} f_{k}^{a} \cdot r_{k}^{a}
$$

where $f_{k}^{a}= \pm 1$ independently for each coordinate $a$ and particle $k$ at position $r_{k}^{a}$. The response function of the system at time $t+t_{w}$ is defined by

$$
R\left(t+t_{w}, t_{w}\right)=\left.\frac{1}{3 N} \sum_{a=1}^{3} \sum_{k=1}^{N}\left\langle\frac{\partial f_{k}^{a} \cdot r_{k}^{a}\left(t+t_{w}\right)}{\partial \epsilon\left(t_{w}\right)}\right\rangle\right|_{\epsilon=0}
$$

The linear regime is probed for small enough values of the perturbation strength $\epsilon$. It is actually more convenient to look at the integrated response function:

$$
\chi\left(t+t_{w}, t_{w}\right)=\int_{t_{w}}^{t+t_{w}} R\left(t+t_{w}, \tau\right) \epsilon(\tau) d \tau .
$$

In order to measure this quantity we prepare the system in a non-equilibrium state by quenching to the subcritical value of $1 / \mu$ and letting it relax up to time $t_{w}$; at $t_{w}$, we make a copy of the system and apply the perturbation; we then let evolve the two systems with the same succession of random numbers and measure the difference $\Delta r_{k}^{a}(t+$ $\left.t_{w}\right)$ between the displacements that take place in the two systems at time $t+t_{w}$. At constant field we obtain

$$
\chi\left(t+t_{w}, t_{w}\right)=\frac{1}{3 N} \sum_{a=1}^{3} \sum_{k=1}^{N}\left\langle f_{k}^{a} \cdot \Delta r_{k}^{a}\left(t+t_{w}\right)\right\rangle .
$$

We have performed a sudden quench to the subcritical value $1 / \mu=1 / 2.2$ starting from a configuration with density 0.75 and measured the integrated response function $\chi\left(t+t_{w}, t_{w}\right)$ vs. time $t$ at different waiting times $t_{w}$. We have checked that for $0.05<\epsilon<0.15$ non-linear effects are absent. In the following we present the results for the case $\epsilon=0.1$ and for a cubic lattice of size $20^{3}$. Figure 1 shows that for small values of $t_{w}$ TTI holds, and aging effects set in only for larger value of $t_{w}$. To obtain the scaling behavior one has to consider an effective waiting time, $\tau_{w}=t_{w}+\tau_{0}$, that takes into account the relaxation time, $\tau_{0}$, of the system before the quench. Indeed, figure 2 shows that, if one plots $\chi\left(t+t_{w}, t_{w}\right)$ vs. $t / \tau_{w}$, the curves lie roughly on top of each other and follow a simple aging logarithmic behavior, $\chi\left(t+t_{w}, t_{w}\right) \sim \log \left(1+t / \tau_{w}\right)$. Similar results were previously found also for the mean square displacement 10,11.

Fluctuation-dissipation ratio. We are now in a position to investigate the nature of breakdown of thermodynamical equilibrium. We consider the generalized FDT proposed by Cugliandolo and Kurchan in the framework of dynamical mean-field theory of spherical $p$-spin glass model [12]. In our case the relevant property is the off-equilibrium generalization of Einstein-Stokes relation among diffusion coefficient and viscosity, given by [14]:

$$
X(B) \frac{\partial B\left(t+t_{w}, t_{w}\right)}{\partial t_{w}}=-2 T R\left(t+t_{w}, t_{w}\right),
$$

where

$B\left(t+t_{w}, t_{w}\right)=\frac{1}{3 N} \sum_{a=1}^{3} \sum_{k=1}^{N}\left\langle\left[r_{k}^{a}\left(t+t_{w}\right)-r_{k}^{a}\left(t_{w}\right)\right]^{2}\right\rangle$,

is the mean square displacement. The fluctuationdissipation ratio (FDR), $X$, depends on both times only through $B$ and its departure from 1 is a measure of violation of FDT. The integrated version of the generalized FDT allows to write (considering a constant field):

$$
\chi\left(t+t_{w}, t_{w}\right)=\frac{\epsilon}{2 T} \int_{0}^{B\left(t+t_{w}, t_{w}\right)} X(B) d B .
$$

(We always write explicitly the temperature $T$, however it does not play any role.) Therefore, if FDT holds, we 
would have a straight line with slope $\epsilon / 2 T$ in the parametric plot of $B\left(t+t_{w}, t_{w}\right)$ versus $\chi\left(t+t_{w}, t_{w}\right)$; while a deviation from this straight line indicates a failure of FDT. The way in which FDT breaks down plays a key role in aging systems since it signals the presence of a time-scale dependent effective temperature [13], and this violation can be bounded by a quantity related to the entropy production [16]. The FDR has been measured by Montecarlo simulations in several models such as spinglasses [17]20], Lennard-Jones binary mixtures [18], domain growth models 19], and directed polymers [21], and so far all the findings are consistent with the prediction of generalized FDT. We have carried out extensive numerical simulation to measure the FDR, $X(B)$, in three dimensional lattice-gas models with kinetic constraints. We observe the two asymptotic regimes also found in the out of equilibrium dynamics of mean field models of structural glasses [12]:

- at times $t$ smaller than $t_{w}$ we observe a short-time quasi equilibrium regime where FDT holds;

- at larger separation times FDT breaks down with a constant violation factor.

These results appear clearly in the parametric plot 15] of $\chi\left(t+t_{w}, t_{w}\right)$ vs. $B\left(t+t_{w}, t_{w}\right)$ where, as can be seen in fig. 3, the curve approaches the characteristic broken line. (In order to probe the asymptotic time regime we have chosen $t_{w}=10^{5}$.) It is natural in this context to interpret the quasi-equilibrium regime as corresponding to the motion of particle inside a cage, although the size of cage in this model is quite small and of the order of a fraction of lattice spacing. After that the system crosses over a diffusion-cage regime where a departure from equilibrium is observed with a constant FDR.

In order to see if the inverse chemical potential plays the role of a "true" temperature, we have measured the function $X(B)$ by quenching the system at different subcritical values. Figure 4 shows that the slope of the FDT violating line, as well as the location of the breaking point, are independent of the quench value of $1 / \mu$, at least in the time window explored in our numerical experiments and in the range of values of $1 / \mu$ used. Therefore as suggested in ref. [11], the FDR appears locked in at the value the system would exhibit at the critical density, and should be only related with "universal" properties of the model, like for instance the exponent $\phi$.

Conclusions. To summarize we have shown that three dimensional lattice-gas models defined by short range kinetic constraint and trivial equilibrium Boltzmann-Gibbs measure exhibit a simple aging behavior in the integrated response function and violate fluctuation-dissipation theorem in a manner similar to mean field models of structural glasses. The fluctuation-dissipation ratio in the glassy phase appears to be a constant independent on the chemical potential of the reservoir; however, different results are expected if the boundary condition is changed or if creation and destruction of particles within the bulk is allowed.

Given the non-holonomic nature of kinetic constraints and the trivial hamiltonian of the model, any statistical mechanics approach based on the calculation of some partition function should be unsuitable to grasp the features of the glassy phase. In order to understand if aging is interrupted after a certain characteristic time [22], it would be interesting to analytically investigate the longtime dynamical behavior of the model. A persistent aging scenario would prove that also in finite-dimensional short-range models the glass transition may have a purely dynamic origin unrelated to any underlying thermodynamic singularity.

\section{ACKNOWLEDGMENTS}

This work was originated by inspiring collaboration with Jorge Kurchan and Luca Peliti. I wish to thank them for several key suggestions and discussions. I am grateful to Jeferson J. Arenzon and Cláudia P. Nunes for the kind hospitality in Porto Alegre where this work was completed.

[1] L.C.E. Struik, Physical Aging in Amorphous Polymers and Other Materials (Amsterdam: Elsevier, 1978).

[2] J. Zarzycki, Glasses and vitreous state (Cambridge: Cambridge University Press, 1991)

[3] W. Götze, in Liquids, freezing and glass transition, Les Houches 1989, edited by J.-P. Hansen, D. Levesque, J. Zinn-Justin (Amsterdam: North Holland, 1991).

[4] For a review, see: J.-P. Bouchaud, L.F. Cugliandolo, J. Kurchan and M. Mézard, in Spin Glasses and Random Fields, edited by P. Young, (Singapore: World Scientific, 1998), and references therein.

[5] S.P. Das and G.F. Mazenko Phys. Rev. A 342265 (1986).

[6] T.R. Kirkpatrick and D. Thirumalai Phys. Rev. B 36 5388 (1987).

[7] R. Monasson Phys. Rev. Lett. 752847 (1995).

[8] S. Franz and G. Parisi, Phys. Rev. Lett. 792486 (1997).

[9] W. Kob and H.C. Andersen, Phys. Rev. E 484364 (1993).

[10] J. Kurchan, L. Peliti and M. Sellitto, Europhys. Lett. 39 365 (1997).

[11] L. Peliti and M. Sellitto, cond-mat 9702221; to appear in Disorder and Chaos edited by A. Vulpiani, Editions de Physique (1998).

[12] L.F. Cugliandolo and J. Kurchan, Phys. Rev. Lett. 71 173 (1993); Phil. Magaz. B 7150 (1995).

[13] L.F. Cugliandolo, J. Kurchan and L. Peliti Phys. Rev. E 553898 (1997). 
[14] L.F. Cugliandolo and P. Le Doussal, Phys. Rev. E 53 1525 (1996).

[15] L.F. Cugliandolo and J. Kurchan J. Phys. A 275749 (1994).

[16] L.F. Cugliandolo, D. Dean and J. Kurchan Phys. Rev. Lett. 792168 (1997).

[17] S. Franz and H. Rieger, J. Stat. Phys. 79749 (1995).

[18] G. Parisi Phys. Rev. Lett. 793660 (1997).

[19] A. Barrat, cond-mat 9710069.

[20] E. Marinari, G. Parisi, F. Ricci-Tersenghi and J.J. RuizLorenzo, cond-mat 9710120.

[21] H. Yoshino, cond-mat 9802283.

22] S. Franz, M. Mezard, G. Parisi and L. Peliti, cond-mat 9803108 .

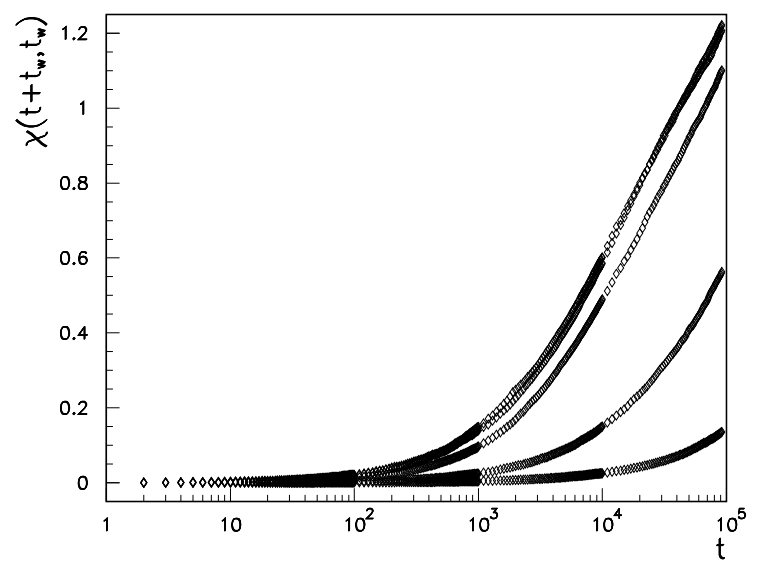

FIG. 1. Integrated response function $\chi\left(t+t_{w}, t_{w}\right)$ vs. time $t$ after a quench to the subcritical values $1 / \mu=1 / 2.2$. The measurements were carried out after the perturbation was applied at waiting times $t_{w}=10,10^{2}, 10^{3}, 10^{4}, 10^{5}$. The initial density is 0.75 . Average over 20 samples.

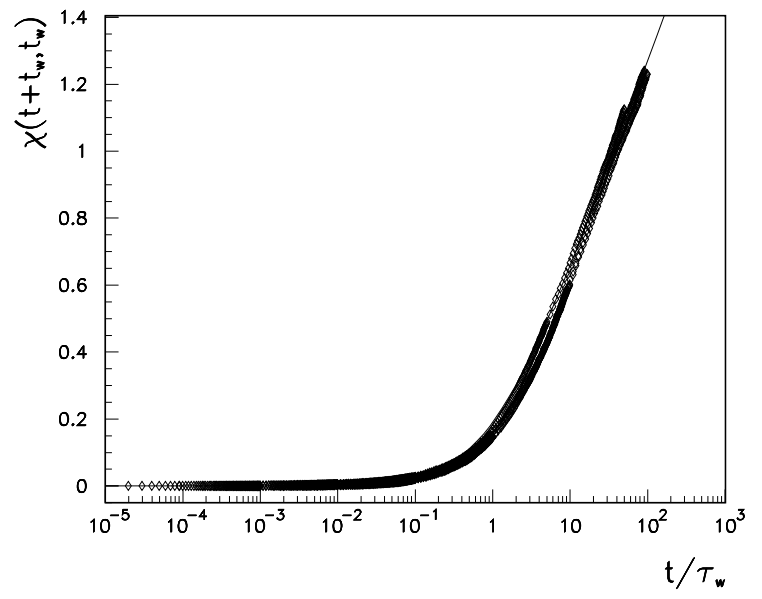

FIG. 2. Scaling behavior of integrated response function $\chi\left(t+t_{w}, t_{w}\right)$ vs. $t / \tau_{w}$, where $\tau_{w}=t_{w}+\tau_{0}$ with $\tau_{0}=10^{3}$ and $t_{w}=10,10^{2}, 10^{3}, 10^{4}, 10^{5}$. The full line corresponds to $\chi\left(t+t_{w}, t_{w}\right) \sim \log \left(1+t / \tau_{w}\right)$.

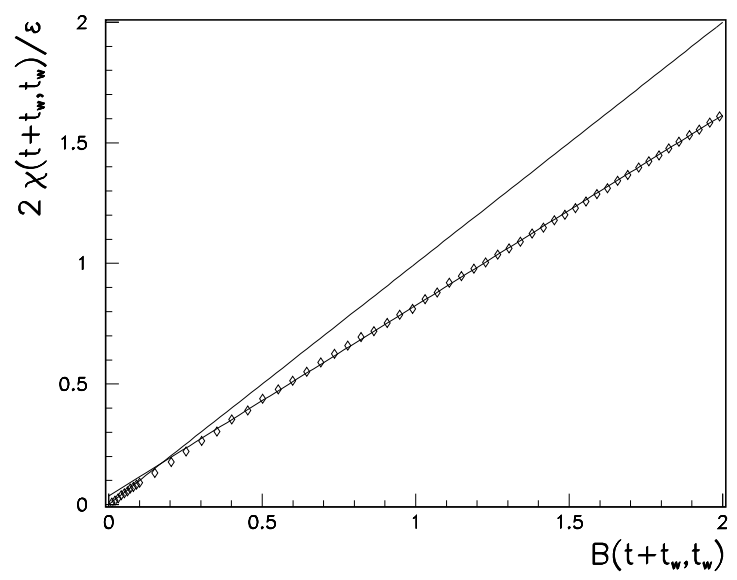

FIG. 3. Parametric plot of $2 \chi\left(t+t_{w}, t_{w}\right) / \epsilon$ vs. mean square displacement $B\left(t+t_{w}, t_{w}\right)$ measured after a waiting time $t_{w}=10^{5}$ from a quench to the subcritical value $1 / \mu=1 / 2.2$. The density of initial configuration is 0.75 . The two straight line have slope 1 (FDT line) and 0.79. Average over 200 samples; error bars comparable to symbol size.

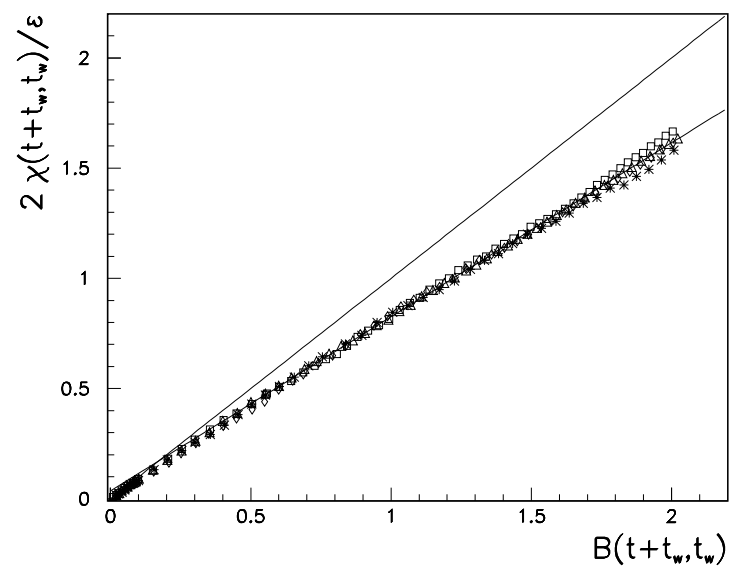

FIG. 4. Parametric plot of $2 \chi\left(t+t_{w}, t_{w}\right) / \epsilon$ vs. mean square displacement $B\left(t+t_{w}, t_{w}\right)$ measured after a waiting time $t_{w}=10^{5}$ from a quench to the subcritical value $1 / \mu=1 / 2.05$ (stars), 1/2.1 (triangles), 1/2.2 (diamonds), 1/2.3 (squares). The density of initial configuration is 0.75 . The two straight line have slope 1 and 0.79. Average over 200 samples; error bars comparable to symbols size. 